\title{
On the branching convolution equation $\mathcal{E}=\mathcal{Z} \circledast \mathcal{E}$
}

\author{
Pascal Maillard* $\quad$ Bastien Mallein ${ }^{\dagger \neq}$
}

\begin{abstract}
We characterize all random point measures which are in a certain sense stable under the action of branching. Denoting by $\circledast$ the branching convolution operation introduced by Bertoin and Mallein, and by $\mathcal{Z}$ the law of a random point measure on the real line, we are interested in solutions to the fixed-point equation $\mathcal{E}=\mathcal{Z} \circledast \mathcal{E}$, with $\mathcal{E}$ a random point measure distribution. Under suitable assumptions, we characterize all solutions of this equation as shifted decorated Poisson point processes with a uniquely defined shift.
\end{abstract}

Keywords: Branching random walk; branching convolution operation; point process; extremal process; smoothing transform; fixed-point equation.

MSC2020 subject classifications: Primary 60G55; 60J80; 60G70, Secondary 60G42; 60G50.

Submitted to ECP on June 5, 2021, final version accepted on September 24, 2021.

Supersedes arXiv: 2106.02544.

Supersedes HAL: hal - 03251326.

\section{Introduction}

In the recent years, the asymptotic behaviour of extremal particles in branching processes has been the subject of a large literature. Starting from the results of Bramson [14] and Lalley and Sellke [19] on the asymptotic behaviour of the maximum of branching Brownian motions, the convergence in distribution of the position of the largest atom in branching random walks was obtained by Aïdékon [4]. In both cases, the limiting distribution was identified as a randomly shifted Gumbel distribution.

More generally, the convergence in distribution of the extremal process and the study of the limit has been a recurrent subject of interest in the last decade. This convergence was obtained for branching Brownian motion by Aïdékon et al. [1] and Arguin et al. [2]. Madaule [21], relying on [22], extended this convergence result to branching random walks: the extremal process of the branching random walk converges to a randomly shifted Poisson point process with exponential intensity, decorated by i.i.d. copies of a random point measure. This limiting point process was referred to in [25] as a randomly Shifted Decorated Poisson Point Process, or SDPPP.

The convergence of extremal processes to SDPPP has since been observed in a variety of processes presenting a branching-type structure. Among these results, we can mention

\footnotetext{
*Institut de Mathématiques de Toulouse, CNRS, UMR5219, Université de Toulouse, 118 route de Narbonne, 31062 Toulouse cedex 09, France. E-mail: pascal.maillard@math.univ-toulouse. $\mathrm{fr}$

${ }^{\dagger}$ LAGA, UMR 7539, Université Sorbonne Paris Nord, 99 avenue Jean-Baptiste Clément, F-93430, Villetaneuse, France. E-mail: mallein@math . univ-paris13.fr

${ }^{\ddagger}$ DMA, UMR 8553, École Normale Supérieure, 45 rue d’ULM, F-75005, Paris.
} 
- time-inhomogeneous branching Brownian motions [6, 23], in which for a process of length $t \in \mathbb{R}_{+}$, the variance of particles at time $s \leq t$ depends continuously on $s / t$,

- generalized Random Energy Model [24], in which particles branch for $n^{\alpha}$ steps before displacing independently for $n^{\alpha}$ steps,

- discrete Gaussian free field on the square [10], which is a Gaussian field on $\mathbb{Z}^{2} \cap[0, N]$ with correlation given by the Green kernel of the random walk killed when hitting the boundary,

- catalytic branching Brownian motion [13] in which particles move as i.i.d. Brownian motions but can only branch in a neighbourhood of the origin,

- randomly decorated branching random walks [5], in which the extremal process of a branching random walk is decorated by i.i.d. random variables with exponential tail,

- reducible multitype branching Brownian motion [12], in which two types of particles are present, particles of type 1 giving birth to particles of type 2 but not reciprocally.

In all cases the limit of the suitably translated extremal process has been proved to converge to an SDPPP. The aim of this article is to present a unifying theory. We consider random point measures which are in a certain sense stable under the action of branching. We characterize these processes as SDPPP with an explicit random shift. As argued below, such a characterization should allow to streamline proofs of convergence of extremal processes of branching-type systems.

\subsection{Point measures and branching random walks}

We denote by $\mathfrak{P}$ the set of point measures on $\mathbb{R}$ assigning finite mass to the interval $(0, \infty)$. Specifically, $D \in \mathfrak{P}$ if and only if $D$ is a measure on $\mathbb{R}$ with an integer-valued tail, i.e. $\bar{D}(x):=D((x, \infty)) \in \mathbb{Z}_{+}$for every $x \in \mathbb{R}$. Given $D \in \mathfrak{P}$ we call the ranked sequence of atoms of $D$ the non-increasing sequence $\mathbf{d}=\left(d_{n}, n \in \mathbb{N}\right)$ of the positions of the atoms of $D$, repeated according to their multiplicity. In order to account for finite point measures, we write $d_{n}=-\infty$ if $D(\mathbb{R})<n$. In the rest of this article, we canonically identify the point measure $D$ with its ranked sequence of atoms $\mathbf{d}$ via

$$
D=\sum_{n=1}^{\infty} \mathbb{1}_{\left\{d_{n}>-\infty\right\}} \delta_{d_{n}} \Longleftrightarrow \mathbf{d}=(\sup \{y \in \mathbb{R}: D((y, \infty))<n\}, n \in \mathbb{N})
$$

Therefore we canonically identify $\mathfrak{P}$ with the space of non-increasing sequences in $[-\infty, \infty)$ such that $\lim _{n \rightarrow \infty} d_{n}=-\infty$, with the identification $\mathbf{d}=D$. In particular, the null measure is identified with the sequence $(-\infty,-\infty, \cdots)$, and a Dirac mass at point $a \in \mathbb{R}$ with $(a,-\infty,-\infty, \ldots)$. We canonically endow the space of point measures with the smallest $\sigma$-field such that the functions $D \mapsto d_{j}$ are measurable for all $j \in \mathbb{N}$.

Given $D \in \mathfrak{P}$ and $f$ a measurable non-negative function, we set

$$
\langle D, f\rangle=\int f \mathrm{~d} D=\sum_{n=1}^{\infty} \mathbb{1}_{\left\{d_{n}>-\infty\right\}} f\left(d_{n}\right) .
$$

We also denote by $\tau$ the translation operator on $\mathfrak{P}$, defined for all $y \in[-\infty, \infty)$ via

$$
\tau_{y} D=\tau_{y} \mathbf{d}=\left(d_{n}+y, n \in \mathbb{N}\right) .
$$

The law of a random point measure $E$ (i.e. a random element of $\mathfrak{P}$ ) is characterized by its Laplace functional (see e.g. [17, Section 9.4]), defined as $\varphi \mapsto \mathbb{E}(\exp (-\langle E, \varphi\rangle))$, defined for $\varphi \in \mathfrak{T}$ a large enough set of test functions. In this article, we denote by $\mathfrak{T}$ the 
set of continuous non-negative bounded functions on $\mathbb{R}$ with support bounded on the left that are not constant.

A branching random walk is a $\mathfrak{P}$-valued stochastic process $\left(Z_{n}, n \geq 0\right)$, starting from $Z_{0}=\delta_{0}$, that satisfies the branching property, i.e. such that for all $n, m$

$$
Z_{n+m} \stackrel{(d)}{=} \sum_{k=1}^{\infty} \tau_{z_{k}} Z_{m}^{(k)} \text {, where }\left(z_{1}, z_{2} \ldots\right) \text { is the ranked sequence of atoms of } Z_{n},
$$

and $\left(Z_{m}^{(k)}, k \in \mathbb{N}\right)$ are i.i.d. copies of $Z_{m}$, further independent of $Z_{n}$. The law of the branching random walk can then be described by recurrence using only the law of $Z:=Z_{1}$ on $\mathfrak{P}$, that we denote by $\mathcal{Z}$. A branching random walk can be described as a particle system on $\mathbb{R}$ starting from a single particle at the origin at time 0 . At each generation, every particle creates offspring around its position, according to an independent point measure of law $\mathcal{Z}$, translated by its position.

Note that in this article, we do not study branching random walks per se, rather, they appear as a tool in the study of point measure distributions which are in a sense stable under the action of branching.

To ensure the well-definedness of the branching random walk $\left(Z_{n}\right)_{n \geq 0}$ (i.e. that $Z_{n} \in \mathfrak{P}$ for all $n \in \mathbb{N}$ a.s.), one usually further requests the non-degeneracy of the Laplace transform of the intensity measure of $Z$. More precisely, we assume the existence of $\theta>0$ such that

$$
\kappa(\theta):=\log \int\left\langle Z, \exp _{\theta}\right\rangle \mathcal{Z}(\mathrm{d} Z)=\log \mathbb{E}\left(\sum_{j=1}^{\infty} e^{\theta z_{j}}\right)<\infty,
$$

writing $\exp _{\theta}: z \mapsto e^{\theta z}$. Under this assumption and the branching property, one straightforwardly obtains that for all $n \in \mathbb{N}, \mathbb{E}\left(\left\langle Z_{n}, \exp _{\theta}\right\rangle\right)=e^{n \kappa(\theta)}$, and

$$
\left(\left\langle Z_{n}, \exp _{\theta}\right\rangle e^{-n \kappa(\theta)}, n \geq 0\right) \text { is a non-negative martingale. }
$$

The branching property (1.1) can be written more concisely using the notion of branching convolution operation $\circledast$, which acts on probability distributions on $\mathfrak{P}$. The branching convolution operation was introduced in [11] and some of its properties studied there. This operation is an extension to probability distributions on $\mathfrak{P}$ of the usual convolution operation for measures on $\mathbb{R}$. Given $\mathcal{D}$ and $\mathcal{E}$ two probability distributions on $\mathfrak{P}$, their convolution $\mathcal{D} \circledast \mathcal{E}$ is defined as the law of the point measure $\sum_{j=1}^{\infty} \tau_{d_{j}} E^{(j)}$, where $\left(d_{j}, j \geq 1\right)$ is the ranked sequence of atoms of a point measure of law $\mathcal{D}$ and $\left(E^{(j)}\right)$ are i.i.d. random point measures with law $\mathcal{E}$. It is easy to check that the branching convolution operation is associative, and that the law of the Dirac measure $\delta_{0}$ (i.e. the measure $\delta_{\delta_{0}}$ ) is an identity. Denoting by $\mathcal{Z}_{n}$ the law of $Z_{n}$ and by $\mathcal{Z}$ the law of $Z=Z_{1}$, we then have $\mathcal{Z}_{n}=\mathcal{Z}^{\circledast n}=\mathcal{Z}_{n-1} \circledast \mathcal{Z}=\mathcal{Z} \circledast \mathcal{Z}_{n-1}$.

Notation conventions. In this article, we use as much as possible the following typographic convention when dealing with random point measures:

- calligraphic capital letters $\mathcal{D}, \mathcal{Z}, \ldots$ are reserved for laws of a random point measures, i.e. probability distributions on $\mathfrak{P}$;

- roman upper case letters $D, Z, \ldots$ are used to represent random point measures with corresponding distribution, i.e. random variables in the space $\mathfrak{P}$ with law $\mathcal{D}$;

- roman lower case $d_{1}, z_{2}, \ldots$ is used to denote the positions of the largest, second largest, etc. atoms of the point measure $D$;

- boldface lower case $\mathbf{d}=\left(d_{n}, n \in \mathbb{N}\right), \mathbf{z}, \ldots$ is used for the ranked sequence of atoms. 


\subsection{Shifted decorated Poisson point processes}

Given $S$ a non-negative random variable, $\alpha>0$ and $\mathcal{D}$ a law on $\mathfrak{P}$, an SDPPP with parameters $\left(S, e^{-\alpha x} \mathrm{~d} x, \mathcal{D}\right)$ is constructed as follows. Let $\left(\xi_{j}, j \in \mathbb{N}\right)$ denote the ranked sequence of atoms of a Poisson point process with intensity $e^{-\alpha x} \mathrm{~d} x$ and let $\left(D_{i}, i \in \mathbb{N}\right)$ be i.i.d. random point measures with law $\mathcal{D}$. The $\operatorname{SDPPP}\left(S, e^{-\alpha x} \mathrm{~d} x, \mathcal{D}\right)$ is the random point measure defined as

$$
E=\sum_{i=1}^{\infty} \tau_{\alpha^{-1} \log S+\xi_{i}} D_{i}
$$

provided that $E \in \mathfrak{P}$ almost surely. Using the branching convolution operation, the law $\mathcal{E}$ of the SDPPP defined in (1.4) can be written as

$$
\mathcal{E}=\mathcal{S} \circledast \mathcal{X} \circledast \mathcal{D},
$$

where $\mathcal{S}$ is the law of the random point measure $\delta_{\alpha^{-1}} \log S$ and $\mathcal{X}$ is the law of the Poisson process $\left(\xi_{j}, j \in \mathbb{N}\right)$. Remark that convolving on the left with the law of a (random) Dirac measure precisely amounts to a shift by the position of the atom of this Dirac measure. A systematic study of SDPPP was undergone in [25], we recall some of the properties of these random point measures below.

The Laplace functional of an SDPPP $E$ with parameters $\left(S, e^{-\alpha x} \mathrm{~d} x, \mathcal{D}\right)$ can be written

$$
\mathbb{E}\left(e^{-\langle E, \varphi\rangle}\right)=\mathbb{E}\left(\exp \left(-S \int_{\mathbb{R}} e^{-\alpha x}\left(1-e^{-\Psi_{\varphi}(x)}\right) \mathrm{d} x\right)\right),
$$

where $\Psi_{\varphi}(x)=-\log \mathbb{E}\left(e^{-\left\langle\tau_{x} D, \varphi\right\rangle}\right)$ and $D$ is a random point measure with law $\mathcal{D}$. Subag and Zeitouni proved in particular that a characteristic property of SDPPP is the existence of a non-increasing function $g$ such that for every non-null continuous non-negative compactly supported function $\varphi$, there exists $T_{\varphi} \in \mathbb{R}$ such that

$$
\forall y \in \mathbb{R}, \quad \mathbb{E}\left(e^{-\left\langle\tau_{y} E, \varphi\right\rangle}\right)=\mathbb{E}\left(e^{-\langle E, \varphi(\cdot+y)\rangle}\right)=g\left(y-T_{\varphi}\right) .
$$

This result is stated more precisely in Theorem A below. The reader can easily check that for the SDPPP given above, the function $g(y)=\mathbb{E}\left[\exp \left(-S e^{\alpha y}\right)\right]$ satisfies these conditions.

Formula (1.4) is well-defined if and only if the law of $d_{1}=$ : $\max D$, the position of the largest atom in $D$, has an exponential moment of order $\alpha$, i.e.

$$
c:=\mathbb{E}\left(e^{\alpha d_{1}}\right)=\int_{\mathfrak{P}} e^{\alpha \max D} \mathcal{D}(\mathrm{d} D)<\infty .
$$

Indeed, writing $d_{1}^{(i)}=\max D_{i}$ for all $i \in \mathbb{N}$ and recalling that $\left(\xi_{i}, i \in \mathbb{N}\right)$ are the atoms of the Poisson point process, we straightforwardly check by Campbell's formula that (1.8) is equivalent to $\#\left\{i \in \mathbb{N}: \alpha^{-1} \log S+\xi_{i}+d_{1}^{(i)}>y\right\}<\infty$ a.s. for all $y \in \mathbb{R}$. Therefore, $E$ is a random element of $\mathfrak{P}$ if and only if (1.8) holds.

If (1.8) holds, we define the law $\mathcal{D}^{*}$ on $\mathfrak{P}$ by

$$
\int_{\mathfrak{P}} f(D) \mathcal{D}^{*}(\mathrm{~d} D)=\frac{\int_{\mathfrak{P}} f\left(\tau_{-\max D} D\right) e^{\alpha \max D} \mathcal{D}(\mathrm{d} D)}{\int_{\mathfrak{P}} e^{\alpha \max D} \mathcal{D}(\mathrm{d} D)}
$$

for all measurable bounded functions $f$. Remark that the law $\mathcal{D}^{*}$ is supported by $\mathfrak{P}^{*}:=\left\{\mathbf{x} \in \mathfrak{P}: x_{1}=0\right\}$, and $E$ is also an $\operatorname{SDPPP}\left(c S, e^{-\alpha x} \mathrm{~d} x, \mathcal{D}^{*}\right)$, as one readily checks. In other words, we can assume without loss of generality that the law of the decoration of an SDPPP is supported on $\mathfrak{P}^{*}$. 
Under some integrability conditions on $S$ (namely (2.2) below), one can recover the law $\mathcal{D}^{*}$ from the law of $E$ via the following formula

$$
\int_{\mathfrak{P}} f(\mathbf{x}) \mathcal{D}^{*}(\mathrm{~d} \mathbf{x})=\lim _{z \rightarrow \infty} \mathbb{E}\left(f\left(\tau_{-\max E} E\right) \mid \max E \geq z\right),
$$

where $\max E$ is the position of the largest atom in $E$. Additionally,

$$
\mathbb{P}(\max E \leq z)=\mathbb{P}\left(c S+\max _{j \in \mathbb{N}} \xi_{j} \leq x\right)=\mathbb{E}\left(\exp \left(-c S e^{-\alpha z}\right)\right),
$$

using that $\xi_{1}=\max _{j \in \mathbb{N}} \xi_{j}$ follows the Gumbel distribution. As a result, given the value of $\alpha$, both the law of $c S$ and the law of $\mathcal{D}^{*}$ can be identified from the law of $E$.

\subsection{Fixed points of a branching convolution equation}

In this section, we introduce branching convolution equations and state the main result of this article. Writing $\left(z_{n}, n \geq 1\right)$ for the ranked sequence of atoms of $Z$, we assume there exists $\alpha>0$ such that

$$
\mathbb{E}\left(\sum_{j=1}^{\infty} \mathbb{1}_{\left\{z_{j}>-\infty\right\}}\right)>1 \text { and } \mathbb{E}\left(\sum_{j=1}^{\infty} e^{\alpha z_{j}}\right)=1 .
$$

The first condition ensures that $\mathbb{P}\left(\forall n \in \mathbb{N}, Z_{n} \neq 0\right)>0$, i.e. the survival of the branching random walk with positive probability, and the second one that the process $\left(\left\langle Z_{n}, \exp _{\alpha}\right\rangle, n \geq 1\right)$ is a non-negative martingale, that we refer to as the additive martingale. Next, we assume one of the two following conditions holds

$$
\begin{gathered}
\mathbb{E}\left(\sum_{j=1}^{\infty} z_{j} e^{\alpha z_{j}}\right) \in(-\infty, 0) \text { and } \mathbb{E}\left(\left\langle Z, \exp _{\alpha}\right\rangle \log _{+}\left\langle Z, \exp _{\alpha}\right\rangle\right)<\infty \\
\mathbb{E}\left(\sum_{j=1}^{\infty} z_{j} e^{\alpha z_{j}}\right)=0, \quad \mathbb{E}\left(\sum_{j=1}^{\infty} z_{j}^{2} e^{\alpha z_{j}}\right)<\infty \\
\text { and } \mathbb{E}\left(\left\langle Z, \exp _{\alpha}\right\rangle\left(\log _{+}\left\langle Z, \exp _{\alpha}\right\rangle\right)^{2}\right)+\mathbb{E}\left(\tilde{X} \log _{+} \tilde{X}\right)<\infty
\end{gathered}
$$

where $\tilde{X}=\sum_{j=1}^{\infty}\left(z_{j}\right)_{+} e^{\alpha z_{j}}$. If assumption (A2a) holds, we say that we are in the regular case, in opposition with the boundary case when (A2b) holds, following the terminology of [9].

Under assumption (A2a), the martingale $\left\langle Z_{n}, \exp _{\alpha}\right\rangle$ is uniformly integrable and converges a.s. to a non-degenerate limit that we denote by $S$ (see Biggins [7], Lyons [20]), whereas under assumption (A2b), it converges to 0 almost surely. In that situation, writing $f: x \mapsto x e^{\alpha x}$, the process $\left(\left\langle Z_{n}, f\right\rangle\right)$ is a (signed, non-uniformly integrable) martingale converging almost surely to a non-degenerate, non-negative limit that we also denote by $S$ (see [4], [16]). This martingale is called the derivative martingale. We observe that in both cases, the variable $S$ satisfies

$$
S \stackrel{(d)}{=} \sum_{j=1}^{\infty} e^{\alpha z_{j}} S^{(j)}
$$

where $\left(S^{(j)}, j \in \mathbb{N}\right)$ are i.i.d. copies of $S$ that are further independent of $Z$.

In the rest of the article, we assume

$$
\text { (A2a) or (A2b) holds, }
$$


and we write $S$ the limit of the additive or the derivative martingale depending on whether we are in the regular or the boundary case. Finally, we assume the branching random walk to be non-lattice, i.e.

$$
\forall a>0, \forall b \in \mathbb{R}, \mathbb{P}\left(\forall j \in \mathbb{N}, z_{j} \in a \mathbb{Z}+b\right)<1 .
$$

Under assumptions (A1), (A2) and (A3) we take interest in random point measures $E$ satisfying the following equality in distribution

$$
E \stackrel{(d)}{=} \sum_{j=1}^{\infty} \tau_{z_{j}} E^{(j)}
$$

where $\left(E^{(j)}, j \in \mathbb{N}\right)$ are i.i.d. copies of $E$ further independent of $\mathbf{z}=Z$.

Then, denoting by $\mathcal{Z}$ the law of the first generation of the branching random walk, we can reformulate the problem of identifying the solutions $E$ to the equation in law (1.13) as the identification of probability measures $\mathcal{E}$ on $\mathfrak{P}$ satisfying

$$
\mathcal{E}=\mathcal{Z} \circledast \mathcal{E} .
$$

The main result of the article is the following characterization of the solutions of this fixed-point equation.

Theorem 1.1. Under assumptions (A1), (A2) and (A3), a random point measure $E$ satisfies (1.13) if and only if there exist $c>0$ and a probability distribution $\mathcal{D}^{*}$ on $\mathfrak{P}^{*}$ such that $E$ is a $\operatorname{SDPPP}\left(c S, e^{-\alpha x} \mathrm{~d} x, \mathcal{D}^{*}\right)$.

Equivalently, Theorem 1.1 can be reformulated as follows: under assumptions (A1), (A2) and (A3) a distribution $\mathcal{E}$ on $\mathfrak{P}$ satisfies (1.13) if and only if there exists a positive constant $c$ and a probability distribution $\mathcal{D}^{*}$ on $\mathfrak{P}^{*}$, such that $\mathcal{E}=\mathcal{S}_{c} \circledast \mathcal{X} \circledast \mathcal{D}^{*}$, where $\mathcal{S}_{c}$ is the law of the random point measure $\delta_{\alpha^{-1}} \log (c S)$ and $\mathcal{X}$ is the law of a Poisson process with intensity $e^{-\alpha x} \mathrm{~d} x$.

Remark 1.2. Note that if $\mathcal{E}$ is a point measure distribution satisfying (1.14), then for any probability distribution $\mathcal{F}$ on $\mathfrak{P}^{*}$, the point measure defined as $\overline{\mathcal{E}}=\mathcal{E} \circledast \mathcal{F}$ also satisfies (1.14), by associativity of $\circledast$. Indeed, we have $\mathcal{Z} \circledast \overline{\mathcal{E}}=\mathcal{Z} \circledast \mathcal{E} \circledast \mathcal{F}=\mathcal{E} \circledast \mathcal{F}=\overline{\mathcal{E}}$. As a consequence, satisfying (1.14) alone is not enough to characterize the law $\mathcal{D}^{*}$.

Theorem 1.1 can be used to characterize the law of the limit of the extremal point measure of a branching random walk, provided that its convergence was already proved. Using the branching property (1.1) at the first generation, i.e. with $m \in \mathbb{N}$ and $n=1$, then letting $m \rightarrow \infty$, we deduce that the limiting point measure has to satisfy (1.13). For example, if the extremal process of the time-inhomogeneous branching Brownian motion studied in [23] were to converge, the limit would have to be an SDPPP, with the normalization constant $c>0$ and the decoration law $\mathcal{D}$ to be determined.

We conjecture that applying the branching property at its last step, i.e. for $m=1$ and $n \in \mathbb{N}$ would allow to complete the characterization of the SDPPP by identifying the limiting point measure. Hence, we are interested in solutions of the following dual fixed-point equation:

$$
\mathcal{E}=\mathcal{E} \circledast \mathcal{Z},
$$

which can be written as $E \stackrel{(d)}{=} \sum_{i=1}^{\infty} \sum_{j=1}^{\infty} \delta_{e_{i}+z_{j}^{i}}$, where $\mathbf{e}$ is the ranked sequence of atoms of $E$, a point measure of law $\mathcal{E}$, and $\left(\mathbf{z}^{i}, i \in \mathbb{N}\right)$ are the ranked sequences of atoms of i.i.d. copies of $Z$.

Conjecture 1.3. Assume (A1) and (A3) and that either (A2b) or

$$
\mathbb{E}\left(\sum_{j=1}^{\infty} z_{j} e^{\alpha z_{j}}\right) \in(0, \infty) \text { and } \mathbb{E}\left(\left\langle Z, \exp _{\alpha}\right\rangle \log _{+}\left\langle Z, \exp _{\alpha}\right\rangle\right)<\infty
$$


There exists a law $\mathcal{D}^{*}$ on $\mathfrak{P}^{*}$, such that the following holds: a probability distribution $\mathcal{E}$ on $\mathfrak{P}$ satisfies (1.15) if and only if there exists a non-negative random variable $\tilde{S}$ such that $\mathcal{E}$ is the law of an $\operatorname{SDPPP}\left(\tilde{S}, e^{-\alpha x} \mathrm{~d} x, \mathcal{D}^{*}\right)$.

Equivalently, this conjecture states that any point measure distribution $\mathcal{E}$ satisfying $\mathcal{E}=\mathcal{E} \circledast \mathcal{Z}$ can be factorized as $\mathcal{E}=\tilde{\mathcal{S}} \circledast \mathcal{X} \circledast \mathcal{D}^{*}$, with $\mathcal{D}^{*}$ uniquely defined, $\mathcal{X}$ the law of a Poisson process with intensity $e^{-\alpha x} \mathrm{~d} x$, and $\tilde{\mathcal{S}}$ the law of some random shift. If the conjecture holds, then combined with Theorem 1.1, a point measure distribution such that $\mathcal{E}=\mathcal{E} \circledast \mathcal{Z}_{1}=\mathcal{Z}_{2} \circledast \mathcal{E}$ will be uniquely defined, up to translation by a constant.

The conjecture is consistent with the convergence results obtained by Bovier and Hartung [6] and Maillard and Zeitouni [23] for time-inhomogeneous branching Brownian motions. In [6], the limiting point measure satisfies $\mathcal{E}=\mathcal{E} \circledast \mathcal{Z}_{1}=\mathcal{Z}_{2} \circledast \mathcal{E}$ with $\mathcal{Z}_{2}$ verifying (A2a) and $\mathcal{Z}_{1}$ verifying (A2c). In [23] the candidate limit for the extremal process would satisfy similar equalities with both $\mathcal{Z}_{1}$ and $\mathcal{Z}_{2}$ satisfying (A2b).

Conjecture 1.3 has recently been proved by [15] for branching Brownian motions with drift, under the analogue of assumption (A2b). They discuss in this article the possible adaptation of the same techniques to branching random walk settings. Kabluchko [18] obtained convergence results for branching random walks satisfying assumption (A2c) starting from an initial condition given by a Poisson process with exponential intensity.

\section{Proof of Theorem 1.1}

In the rest of the article, $S$ will stand from the limit of the additive or the derivative martingale, depending on whether (A2a) or (A2b) hold, as in Section 1.3. The proof of Theorem 1.1 is decomposed into two largely independent parts: we first show that for all $c>0$ and $\mathcal{D}$ a probability distribution on $\mathfrak{P}^{*}$, the $\operatorname{SDPPP}\left(c S, e^{-\alpha x} \mathrm{~d} x, \mathcal{D}\right)$ is a solution to (1.13), by straightforward computation of its Laplace transform. Then, we show that any solution to (1.13) can be decomposed as a SDPPP, using their characterization in [25].

\subsection{Theorem 1.1: Sufficiency}

As a first step towards the proof of Theorem 1.1, we observe that a randomly shifted Poisson point process is a solution to (1.13).

Lemma 2.1. Under assumptions (A1), (A2) and (A3), for all $c>0$ the law of the Cox process $E$ with intensity $c S e^{-\alpha x} \mathrm{~d} x$ verifies (1.13).

Proof. This result is a simple consequence of the superposability property of Poisson point process. Given $\left(E^{(i)}, i \in \mathbb{N}\right)$ i.i.d. copies of $E$ with intensity $c S^{(i)} e^{-\alpha x} \mathrm{~d} x$ which are further independent of $Z$, we observe that $\sum_{i=1}^{\infty} \tau_{z_{i}} E^{(i)}$ is a Cox process with intensity $\sum_{i=1}^{\infty} c S^{(i)} e^{-\alpha\left(x-z_{i}\right)} \mathrm{d} x=c e^{-\alpha x}\left(\sum S^{(i)} e^{\alpha z_{i}}\right) \mathrm{d} x$. Then as $S$ satisfies (1.12), the proof is complete.

As a corollary, we obtain that any $\operatorname{SDPPP}\left(c S, e^{-\alpha x} \mathrm{~d} x, \mathcal{D}\right)$ satisfies (1.13).

Corollary 2.2. Let $\mathcal{D}$ be a random point measure distribution on $\mathfrak{P}^{*}$ and $c>0$. Under assumptions (A1), (A2) and (A3), an $\operatorname{SDPPP}\left(c S, e^{-\alpha x} \mathrm{~d} x, \mathcal{D}\right)$ satisfies (1.13).

Proof. It is a direct consequence of Remark 1.2. As $\mathcal{P}$ the law of a Cox process with intensity $c S e^{-\alpha x} \mathrm{~d} x$ satisfies (1.14), this is also the case for $\mathcal{E}=\mathcal{P} \circledast \mathcal{D}$ the law of an $\operatorname{SDPPP}\left(c S, e^{-\alpha x} \mathrm{~d} x, \mathcal{D}\right)$. 


\subsection{Theorem 1.1: Necessity}

We consider in this section a random point measure $E$ satisfying (1.13). For any test function $\varphi \in \mathfrak{T}$, we introduce the function

$$
F_{\varphi}: x \mapsto \mathbb{E}\left(e^{-\left\langle\tau_{x} E, \varphi\right\rangle}\right), \quad \text { for } x \in \mathbb{R} .
$$

We recall here the following consequence of [25, Theorem 10(a)].

Theorem A. Assume that for every compactly supported function $\varphi \in \mathfrak{T}$, there exists $T_{\varphi} \in \mathbb{R}$ such that $F_{\varphi}(x)=g\left(x-T_{\varphi}\right)$, where $g: x \in \mathbb{R} \mapsto \mathbb{E}\left(\exp \left(-W e^{\beta x}\right)\right)$, for some $\beta>0$ and $W$ a non-negative random variable. Assume that

$$
\forall y \in \mathbb{R}, \lim _{x \rightarrow-\infty} \frac{1-g(x+y)}{1-g(x)}=e^{\beta y} .
$$

Then, there exists $c>0$ such that $E$ is an $\operatorname{SDPPP}\left(c W, e^{-\beta x} \mathrm{~d} x, \mathcal{D}^{*}\right)$, where $\mathcal{D}^{*}$ is the limit law constructed using the formula (1.10).

Observe that (2.1) can be rephrased in terms of the law of $W$, it corresponds to the condition

$$
\lambda \mapsto-\log \mathbb{E}\left(e^{-\lambda W}\right) \text { is regularly varying at } 0 \text { with index } 1 .
$$

The rest of the section therefore consists in proving that we are under the conditions of application of Theorem A with $W=S$ and $\beta=\alpha$. We link this problem to the characterization of the fixed point to the smoothing transform. Precisely, we say that a measurable $[0,1]$-valued function $f$ is a fixed point of the smoothing transform associated to the point measure $\mathcal{Z}$ if

$$
\forall x>0, f(x)=\mathbb{E}\left(\prod_{j \in \mathbb{N}: z_{j}>-\infty} f\left(x e^{z_{j}}\right)\right) .
$$

This formalism might be better understood observing that if $f$ is the characteristic function of a random variable $Y$, then (2.3) can be rewritten $Y \stackrel{(d)}{=} \sum_{j=1}^{\infty} e^{z_{j}} Y_{j}$, where $\left(Y_{j}, j \in \mathbb{N}\right)$ are i.i.d. copies of $Y$.

The study of fixed points of the smoothing transform under increasingly general conditions has been the subject of a large corpus of studies, we refer to [3] and the references therein for an overview of the literature. We will use the characterization of fixed points of the smoothing transform obtained in that article, which can be rephrased as follows.

Theorem B. Under assumptions (A1), (A2) and (A3), let $f$ be a non-constant fixed point of the smoothing transform, satisfying (2.3). Suppose furthermore that $f$ satisfies one of the two following conditions:

1. $f$ is left-continuous and non-increasing, or

2. $f(0)=1, f(t) \rightarrow 1$ as $t \rightarrow 0$, and $\log \left(1-f\left(e^{-z}\right)\right)$ is uniformly continuous on $[K, \infty)$ for some $K>0$.

Then there exists $h>0$ such that $f(t)=\mathbb{E}\left(e^{-h S t^{\alpha}}\right)$ for all $t>0$.

With these two results in our hands, we can now prove the necessity part of Theorem 1.1. We begin with the following lemma.

Lemma 2.3. Let $\varphi \in \mathfrak{T}$ be non-decreasing. Under assumptions (A1), (A2) and (A3) there exists $T_{\varphi} \in \mathbb{R}$ such that $F_{\varphi}(x)=\mathbb{E}\left(\exp \left(-S e^{\alpha\left(x-T_{\varphi}\right)}\right)\right)$. 
Proof. For all $t \geq 0$, we set $f(t)=F_{\varphi}(\log t)$. Using (1.13), we have

$$
\begin{aligned}
\mathbb{E}\left(\prod_{j \geq 1: z_{j}>-\infty} f\left(t e^{z_{j}}\right)\right) & =\mathbb{E}\left(\exp \left(-\sum_{j \geq 1}\left\langle\tau_{z_{j}+\log t} E^{(j)}, \varphi\right\rangle\right)\right) \\
& =\mathbb{E}\left(\exp \left(-\left\langle\tau_{\log t} \sum_{j \geq 1} \tau_{z_{j}} E^{(j)}, \varphi\right\rangle\right)\right) \\
& =\mathbb{E}\left(\exp \left(-\left\langle\tau_{\log t} E, \varphi\right\rangle\right)\right)=f(t) .
\end{aligned}
$$

Additionally, as $\varphi$ is continuous and non-decreasing, we obtain that $f$ is non-increasing, and continuous by the dominated convergence theorem.

Hence, by Theorem B, there exists $h>0$ such that

$$
f(t)=\mathbb{E}\left(e^{-h S t^{\alpha}}\right)=\mathbb{E}\left(\exp \left(-S e^{\alpha\left(\log t-T_{\varphi}\right)}\right)\right)
$$

setting $T_{\varphi}=(\log h) / \alpha$. This finishes the proof.

For all $x \in \mathbb{R}$, we set

$$
g(x)=\mathbb{E}\left(\exp \left(-S e^{\alpha x}\right)\right) .
$$

We note that $g$ is a continuous and decreasing function. Lemma 2.3 shows that for any non-decreasing function $\varphi \in \mathfrak{T}$, there exists $T_{\varphi} \in \mathbb{R}$ such that for all $z \in \mathbb{R}$, we have $\mathbb{E}\left(\exp \left(-\left\langle\tau_{z} E, \varphi\right\rangle\right)\right)=g\left(t-T_{\varphi}\right)$. We recall the asymptotic behaviour of $g$ as $t \rightarrow 0$.

Theorem C. Under assumptions (A1), (A2) and (A3), we have

$$
g(z)=\left\{\begin{array}{ll}
1-e^{\alpha z}(1+o(1)) & \text { if (A2a) holds } \\
1+\alpha z e^{\alpha z}(1+o(1)) & \text { if (A2b) holds }
\end{array} \quad \text { as } z \rightarrow-\infty\right.
$$

Proof. The asymptotic behaviour of $g(z)$ as $z \rightarrow-\infty$ under assumption (A2a) is a direct consequence of the fact that $\mathbb{E}(S)=1$ as the martingale $\left(\left\langle Z_{n}, \exp _{\alpha}\right\rangle\right)$ is uniformly integrable, see Section 1.3. Under assumption (A2b), we use [8, Theorem 2.1] to compute an equivalent for $1-g(z)$ as $z \rightarrow-\infty$.

Using Lemma 2.3 and Theorem $C$, we can obtain estimates on the law of $E$. In particular, we obtain the law of max $E$.

Proposition 2.4. There exists $c_{m}>0$ such that $\mathbb{P}(\max E \leq x)=\mathbb{E}\left(e^{-c_{m} S e^{-\alpha x}}\right)$ for all $x \in \mathbb{R}$. In particular, $z \mapsto-\log \mathbb{P}(\max E>z)$ is uniformly continuous on $[K, \infty)$ for some $K$ large enough.

Proof. For $\lambda>0$, we set $\varphi_{\lambda}(x)=\max (0, \min (1, \lambda x))$. Applying the monotone convergence theorem, for all $z \in \mathbb{R}$ and $\mu>0$, we have $\lim _{\lambda \rightarrow \infty} F_{\mu \varphi_{\lambda}}(z)=\mathbb{E}\left(e^{-\mu E((-z, \infty))}\right)$. By Lemma 2.3, for all $\lambda, \mu \geq 0$, there exists $T_{\lambda, \mu}$ such that $F_{\mu \varphi_{\lambda}}(z)=g\left(z-T_{\lambda, \mu}\right)$. Indeed, as $g$ is continuous, decreasing and $\lim _{z \rightarrow-\infty} g(z)=1$, we have $T_{\lambda, \mu}=-g^{-1}\left(F_{\mu \varphi_{\lambda}}(0)\right)$. As $g^{-1}$ is continuous, $T_{\lambda, \mu}$ converges, as $\lambda \rightarrow \infty$ to $T_{\infty, \mu}=-g^{-1}\left(\mathbb{E}\left(e^{-\mu E((0, \infty))}\right)\right)$. As a result, we deduce that for all $\mu>0$, we have

$$
\forall z \in \mathbb{R}, \mathbb{E}\left(e^{-\mu E((-z, \infty))}\right)=g\left(z-T_{\infty, \mu}\right) .
$$

Letting $\mu \rightarrow \infty$ and using again the monotone convergence theorem, we have

$$
\forall z \in \mathbb{R}, \mathbb{P}(\max E \leq-z)=g\left(z-T_{\infty, \infty}\right),
$$


with $T_{\infty, \infty}=-g^{-1}(\mathbb{P}(\max E \leq 0))$. We deduce that $\mathbb{P}(\max E \leq x)=\mathbb{E}\left(e^{-c_{m} S e^{-\alpha x}}\right)$, by definition of $g$, where we have set $c_{m}=e^{-\alpha T_{\infty, \infty}}$. Then by Theorem $\mathrm{C}$, we have

$$
-\log (1-g(-z))=\left\{\begin{array}{ll}
\alpha z+o(1) & \text { if (A2a) holds } \\
\alpha z-\log (\alpha z)+o(1) & \text { if (A2b) holds }
\end{array} \quad \text { as } z \rightarrow \infty .\right.
$$

Thus $z \mapsto-\log \mathrm{P}(\max E \geq z)$ is uniformly continuous on $[K, \infty)$, for some $K$, using the Heine-Cantor theorem and the above asymptotic.

Using Lemma 2.3, we also prove that the law of the number of points to the right of $z$ conditioned on $\max E \geq z$ converges as $z \rightarrow \infty$.

Corollary 2.5. There exists a probability measure $\nu$ on $\mathbb{N}$ such that

$$
\forall k \in \mathbb{N}, \quad \lim _{z \rightarrow \infty} \mathbb{P}(E((z, \infty))=k \mid E((z, \infty))>0)=\nu(k) .
$$

Proof. We recall from (2.5) in the proof of Proposition 2.4 that for all $\mu>0$, we have

$$
\forall z \in \mathbb{R}, \mathbb{E}\left(e^{-\mu E((z, \infty))}\right)=g\left(-z-T_{\infty, \mu}\right),
$$

with $T_{\infty, \mu}=-g^{-1}\left(\mathbb{E}\left(e^{-\mu E((0, \infty))}\right)\right)$. As $g$ is decreasing on $\mathbb{R}$, we observe that $T_{\infty, \mu}$ decreases from $\infty$ to $T_{\infty, \infty}$ on $(0, \infty)$.

As a result, we obtain

$$
\mathbb{E}\left(1-e^{-\mu E((z, \infty))} \mid E((z, \infty))>0\right)=\frac{1-g\left(-z-T_{\infty, \mu}\right)}{1-g\left(-z-T_{\infty, \infty}\right)} .
$$

Letting $z \rightarrow \infty$ and using Theorem C, we obtain

$$
\lim _{z \rightarrow \infty} \mathbb{E}\left(1-e^{-\mu E((z, \infty))} \mid E((z, \infty))>0\right)=e^{-\alpha\left(T_{\infty, \mu}-T_{\infty, \infty}\right)} .
$$

It yields

$$
\lim _{z \rightarrow \infty} \mathbb{E}\left(e^{-\mu E((z, \infty))} \mid E((z, \infty))>0\right)=1-e^{-\alpha\left(T_{\infty, \mu}-T_{\infty, \infty}\right)} .
$$

Therefore, the Laplace transform of the law of $E((z, \infty))$, conditionally on $E((z, \infty))>0$, converges pointwise to a continuous function. As a result, we deduce that $E((z, \infty))$ converges in distribution to an integer-valued random variable $X$ with Laplace transform $\mathbb{E}\left(e^{-\mu X}\right)=1-e^{-\alpha\left(T_{\infty, \mu}-T_{\infty}, \infty\right)}$.

We now turn to proving that for any $\varphi \in \mathfrak{T}$, the function $F_{\varphi}(\log t)$ is uniformly continuous in a neighbourhood of $\infty$, so Theorem B can be used again to characterize the Laplace transform $F_{\varphi}$.

Lemma 2.6. Let $\varphi$ be a compactly supported function in $\mathfrak{T}$. Under assumptions (A1), (A2) and (A3) there exists $T_{\varphi} \in \mathbb{R}$ such that

$$
\forall x \in \mathbb{R}, F_{\varphi}(x)=\mathbb{E}\left(\exp \left(-S e^{\alpha\left(x-T_{\varphi}\right)}\right)\right) .
$$

Proof. Without loss of generality, we assume that the support of $\varphi \in \mathfrak{T}$ is a compact subset of $(0, \infty)$. Similarly to the previous proof, we set $f(t)=F_{\varphi}(\log t)$ for all $t>0$. Then with (1.13), we have $f(t)=\mathbb{E}\left(\prod_{j \geq 1: z_{j}>-\infty} f\left(t e^{z_{j}}\right)\right)$, hence $f$ is a fixed point of the smoothing transform. By dominated convergence, $t \mapsto f(t)$ is continuous, hence to apply Theorem B, it is enough to prove that

$$
h: z \mapsto-\log \left(1-f\left(e^{-z}\right)\right)=-\log \mathbb{E}\left(1-e^{-\left\langle\tau_{-z} E, \varphi\right\rangle}\right)
$$


is uniformly continuous on $[K, \infty)$ for some $K>0$.

Using that $\varphi$ has support in $\mathbb{R}_{+}$, for all $z \in \mathbb{R}_{+}$, we have

$$
\mathbb{E}\left(1-e^{-\left\langle\tau_{-z} E, \varphi\right\rangle}\right)=\mathbb{E}\left(1-e^{-\left\langle\tau_{-z} E, \varphi\right\rangle} \mid \max E>z\right) \mathbb{P}(\max E>z) .
$$

Therefore, for all $0 \leq z \leq z^{\prime}$, we have

$$
h(z)-h\left(z^{\prime}\right)=\log \frac{\mathbb{E}\left(1-e^{-\left\langle\tau_{-z^{\prime}} E, \varphi\right\rangle}\right)}{\mathbb{E}\left(1-e^{-\left\langle\tau_{-z} E, \varphi\right\rangle}\right)}=\log \frac{\mathbb{E}\left(1-e^{-\left\langle\tau_{-z^{\prime}} E, \varphi\right\rangle} \mid \max E>z\right)}{\mathbb{E}\left(1-e^{-\left\langle\tau_{-z} E, \varphi\right\rangle} \mid \max E>z\right)}
$$

We note that by Corollary 2.5, we have

$$
\begin{array}{r}
1 \geq \limsup _{z \rightarrow \infty} \mathbb{E}\left(1-e^{-\left\langle\tau_{-z} E, \varphi\right\rangle} \mid \max E>z\right) \geq \liminf _{z \rightarrow \infty} \mathbb{E}\left(1-e^{-\left\langle\tau_{-z} E, \varphi\right\rangle} \mid \max E>z\right) \\
\geq 1-\lim _{z \rightarrow \infty} \mathbb{E}\left(e^{-\|\varphi\|_{\infty} E((z, \infty))} \mid \max E>z\right)>\eta
\end{array}
$$

for some $\eta>0$, using that $\|\varphi\|_{\infty}>0$ as $\varphi \in \mathfrak{T}$.

For all $\delta>0$, we set $\omega(\delta)=\sup _{y \geq 0, y^{\prime} \geq 0,\left|y^{\prime}-y\right|<\delta}\left|\varphi\left(y^{\prime}\right)-\varphi(y)\right|$. We have $\lim _{\delta \rightarrow 0} \omega(\delta)=0$, by uniform continuity of $\varphi$. Then, using the tightness of the law of $E((z, \infty))$ conditionally on $\max E>z$, for all $\varepsilon>0$, there exists $A>0$ such that

$$
\begin{aligned}
& \left|\mathbb{E}\left(1-e^{-\left\langle\tau_{-z^{\prime}} E, \varphi\right\rangle} \mid \max E>z\right)-\mathbb{E}\left(1-e^{-\left\langle\tau_{-z} E, \varphi\right\rangle} \mid \max E>z\right)\right| \\
& \leq \mathbb{E}\left(\left|e^{-\left\langle\tau_{-z^{\prime}} E, \varphi\right\rangle}-e^{-\left\langle\tau_{-z} E, \varphi\right\rangle}\right| \mathbb{1}_{\{E((z, \infty)) \leq A\}} \mid \max E>z\right)+\varepsilon \\
& \leq \mathbb{E}\left(e^{-\left\langle\tau_{-z} E, \varphi\right\rangle}\left|e^{\omega(\delta) E((z, \infty))}-1\right| \mathbb{1}_{\{E((z, \infty)) \leq A\}} \mid \max E>z\right)+\varepsilon \leq\left(e^{\omega(\delta) A}-1\right)+\varepsilon
\end{aligned}
$$

Thus, choosing $\delta>0$ small enough, for all $z, z^{\prime} \in \mathbb{R}_{+}$with $\left|z-z^{\prime}\right| \leq \delta$, (2.7) yields

$$
\left|h\left(z^{\prime}\right)-h(z)\right| \leq\left|\log \left(1-\frac{2 \varepsilon}{\mathbb{E}\left(1-e^{-\left\langle\tau_{-z} E, \varphi\right\rangle} \mid \max E>z\right)}\right)\right| .
$$

As a result, in view of (2.8), there exists $K>0$ large enough such that $\left|h\left(z^{\prime}\right)-h(z)\right| \leq 4 \varepsilon / \eta$ for all $z, z^{\prime}>K$ with $\left|z^{\prime}-z\right|<\delta$. This proves the uniform continuity of $h$. Then, applying Theorem B, there exists $T_{\varphi} \in \mathbb{R}$ such that $F_{\varphi}(z)=g\left(z-T_{\varphi}\right)$ for all $z \in \mathbb{R}$.

Proof of necessity part of Theorem 1.1. Let $E$ be a random point measure satisfying (1.13). By Lemma 2.6, for any $\varphi \in \mathfrak{T}$ with compact support, there exists $T_{\varphi} \in \mathbb{R}$ such that

$$
\mathbb{E}\left(\exp \left(-\left\langle\tau_{z} E, \varphi\right\rangle\right)\right)=\mathbb{E}\left(e^{-S e^{\alpha\left(z-T_{\varphi}\right)}}\right)=g\left(z-T_{\varphi}\right)
$$

By Theorem $\mathrm{C}$, the function $g$ satisfies (2.1), hence by Theorem A, there exists $c>0$ such that $E$ is a $\operatorname{SDPPP}\left(c S, e^{-\alpha x} \mathrm{~d} x, \mathcal{D}^{*}\right)$, with $\mathcal{D}^{*}$ the distribution defined in (1.10).

\section{References}

[1] Elie Aïdékon, Julien Berestycki, Éric Brunet, and Zhan Shi. Branching Brownian motion seen from its tip. Probab. Theory Relat. Fields, 157(1-2):405-451, 2013. MR3101852

[2] Louis-Pierre Arguin, Anton Bovier, and Nicola Kistler. The extremal process of branching Brownian motion. Probab. Theory Relat. Fields, 157(3-4):535-574, 2013. MR3129797

[3] Gerold Alsmeyer, John D. Biggins, and Matthias Meiners. The functional equation of the smoothing transform. Ann. Probab., 40(5):2069-2105, 2012. MR3025711 
On the branching convolution equation $\mathcal{E}=\mathcal{Z} \circledast \mathcal{E}$

[4] Elie Aïdékon. Convergence in law of the minimum of a branching random walk. Ann. Probab., 41(3A):1362-1426, 2013. MR3098680

[5] Antar Bandyopadhyay and Partha Pratim Ghosh. Right-Most Position of a Last Progeny Modified Branching Random Walk. arXiv:2106.02880, jun 2021.

[6] Anton Bovier and Lisa Hartung. Variable speed branching Brownian motion. I: Extremal processes in the weak correlation regime. ALEA, Lat. Am. J. Probab. Math. Stat., 12(1):261291, 2015. MR3351476

[7] John D. Biggins. Martingale convergence in the branching random walk. J. Appl. Probab., 14:25-37, 1977. MR0433619

[8] Dariusz Buraczewski, Alexander Iksanov, and Bastien Mallein. On the derivative martingale in a branching random walk. Ann. Probab., 2020. To appear. MR4255141

[9] John D. Biggins and Andreas E. Kyprianou. Fixed points of the smoothing transform: the boundary case. Electron. J. Probab., 10:609-631, 2005. Id/No 17. MR2147319

[10] Marek Biskup and Oren Louidor. Extreme local extrema of two-dimensional discrete Gaussian free field. Commun. Math. Phys., 345(1):271-304, 2016. MR3509015

[11] Jean Bertoin and Bastien Mallein. Infinitely ramified point measures and branching Lévy processes. Ann. Probab., 47(3):1619-1652, 2019. MR3945755

[12] Mohamed Ali Belloum and Bastien Mallein. Anomalous spreading of reducible multitype branching Brownian motion. arXiv:2011.03223, submitted, nov 2020. MR4254803

[13] Sergey Bocharov. Limiting distribution of particles near the frontier in the catalytic branching Brownian motion. arXiv:1903.07090, submitted, mar 2019. MR4146907

[14] Maury D. Bramson. Maximal displacement of branching Brownian motion. Commun. Pure Appl. Math., 31:531-581, 1978. MR0494541

[15] Xinxin Chen, Christophe Garban, and Atul Shekhar. The fixed points of Branching Brownian Motion. arXiv:2012.03917, submitted, dec 2020.

[16] Xinxin Chen. A necessary and sufficient condition for the nontrivial limit of the derivative martingale in a branching random walk. Adv. in Appl. Probab., 47(3):741-760, 2015. MR3406606

[17] Daryl J. Daley and David Vere-Jones. An introduction to the theory of point processes. Vol. II. Probability and its Applications. Springer, New York, second edition, 2008. MR2371524

[18] Zakhar Kabluchko. Persistence and equilibria of branching populations with exponential intensity. J. Appl. Probab., 49(1):226-244, 2012. MR2952892

[19] Steven P. Lalley and Thomas Sellke. A conditional limit theorem for the frontier of a branching Brownian motion. Ann. Probab., 15:1052-1061, 1987. MR0893913

[20] Russell Lyons. A simple path to Biggins' martingale convergence for branching random walk. In Classical and modern branching processes. Proceedings of the IMA workshop, Minneapolis, MN, USA, June 13-17, 1994, pages 217-221. New York, NY: Springer, 1997. MR1601749

[21] Thomas Madaule. Convergence in law for the branching random walk seen from its tip. $J$. Theor. Probab., 30(1):27-63, 2017. MR3615081

[22] Pascal Maillard. A note on stable point processes occurring in branching Brownian motion. Electron. Commun. Probab., 18:9, 2013. Id/No 5. MR3019668

[23] Pascal Maillard and Ofer Zeitouni. Slowdown in branching Brownian motion with inhomogeneous variance. Ann. Inst. Henri Poincaré, Probab. Stat., 52(3):1144-1160, 2016. MR3531703

[24] Marius Alexander Schmidt and Nicola Kistler. From Derrida's random energy model to branching random walks: from 1 to 3. Electron. Commun. Probab., 20:12, 2015. Id/No 47. MR3358969

[25] Eliran Subag and Ofer Zeitouni. Freezing and decorated Poisson point processes. Comm. Math. Phys., 337(1):55-92, 2015. MR3324155

Acknowledgements. We are grateful to the anonymous referee for the relevant comments that helped improve the first version of this article. 